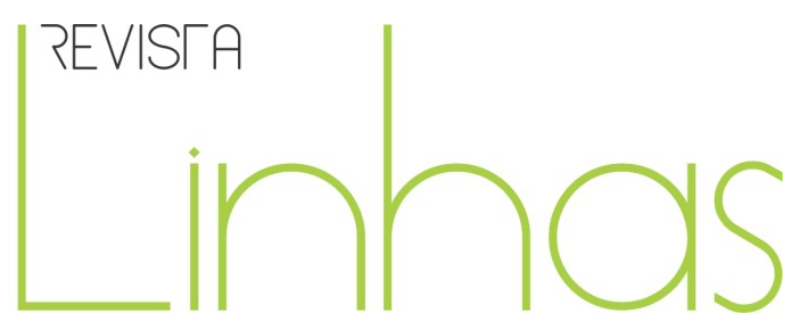

\title{
Retour sur Les Héritiers de Pierre Bourdieu et Jean-Claude Passeron
}

\section{Résumé}

Les Héritiers de Pierre Bourdieu et Jean-Claude Passeron est devenu un «classique » de la sociologie française de l'éducation. L'article présente la place de l'ouvrage dans la sociologie de l'éducation renaissante au début des années 1960, dont une grande partie des travaux portait sur la démocratisation de l'enseignement. L'article analyse aussi les modes de recueil et de traitements des données adoptés par les auteurs des Héritiers. Si le mode de recueil des données apparaît très artisanal, le mode de traitement de celles-ci une certaine innovation par rapport aux recherches antérieures. Les Héritiers caractérise finalement certains des traits de l'histoire de la sociologie française dans les années soixante.

Mots-clés: Les Héritiers; Pierre Bourdieu et Jean-Claude Passeron; Sociologie de l'Education; 1960.

\section{Philippe Masson}

Doutor em Sociologia pela École des Hautes Études en Sciences Sociales - EHESS - Paris/França philippe.masson@univ-nantes.fr

\section{Para citar este artigo:}

MASSON, Philippe. Retour sur Les Héritiers de Pierre Bourdieu et Jean-Claude Passeron. Revista Linhas. Florianópolis, v. 15, n. 29, p. 71-91, jul./dez. 2014.

DOI: $10.5965 / 1984723815292014071$

http://dx.doi.org/10.5965/1984723815292014071 


\section{Back to The Inheritors of Pierre Bourdieu and Jean- Claude Passeron}

\begin{abstract}
Les Héritiers by Pierre Bourdieu et Jean-Claude Passeron has become a classic in French education sociology. This article presents the position of the book in the revived education sociology at the beginning of the 1960s, where a large part of the work focused on the democratization of education. The article analyses too the way data was gathered and processed by the authors of Les Héritiers. If the collection method appears to be very traditional, the processing method introduces some innovation in comparison to previous research. Finally, Les Héritiers presents certain characteristics of the history of French sociology in the 1960 s.
\end{abstract}

Keywords: The Inheritors; Pierre Bourdieu and Jean-Claude Passeron; Sociology of Education; 1960.

\section{Retorno sobre Os herdeiros de Pierre Bourdieu e Jean- Claude Passeron}

\section{Resumo}

Os herdeiros de Pierre Bourdieu e JeanClaude Passeron se tornou um "clássico" da sociologia francesa da educação. Este artigo apresenta o lugar desta obra na sociologia da educação renascente no início dos anos 1960, momento em que grande parte dos trabalhos tratava da democratização do ensino. Nele são analisados os modos de coleta e de tratamento dos dados adotados pelos autores de Os herdeiros. Se o modo de coleta dos dados parece muito artesanal, o modo de tratamento dos mesmos apresenta uma certa inovação em relação às pesquisas anteriores. Os herdeiros revela, finalmente, alguns dos traços da história da sociologia francesa nos anos sessenta.

Palavras-chave: Os Herdeiros; Pierre Bourdieu e Jean-Claude Passeron; Sociologia da Educação; 1960. 
Le livre de Pierre Bourdieu et Jean-Claude Passeron, Les Héritiers, est devenu un classique de la sociologie française ${ }^{1}$. Il s'est vendu à plus de 100000 exemplaires, il est largement connu en dehors du cercle étroit des sociologues et il a eu une influence importante dans la sociologie française comme dans la politique scolaire de ce pays et, plus largement, dans les conceptions que nous avons aujourd'hui sur l'école. Les manuels qui présentent un panorama de la sociologie de l'éducation, voire de l'ensemble de la sociologie française, évoquent fréquemment cet ouvrage pour caractériser le point de vue de ses auteurs sur l'institution scolaire ou pour retracer l'histoire de la spécialité depuis la Seconde guerre mondiale. Il est devenu une référence obligée pour qui souhaite analyser, d'un point de vue sociologique, les étudiants. Il est parfois considéré, avec d'autres ouvrages de Pierre Bourdieu - comme la Distinction que j'évoquerai plus loin comme emblématique d'une école sociologique. Bref, c'est un bon exemple de comptes rendus de recherches empiriques de la sociologie française qui occupe une place importante dans son histoire.

L'ouvrage de Bourdieu et Passeron parait en 1964 dans une période de croissance de la scolarisation dans les enseignements secondaire et supérieur français qui s'accompagne de nombreux débats sur la démocratisation. La formation d'une maind'œuvre qualifiée quantitativement suffisante par rapport aux besoins de l'économie apparaît nécessaire aux planificateurs et aux hommes politiques au pouvoir. Il n'est donc plus acceptable que l'accès aux positions professionnelles qualifiées soit réservé à une petite fraction de la population favorisée par une origine sociale aisée. Dès lors, avec l'arrivée de classes d'âge nombreuses dans l'enseignement secondaire et la mise en œuvre de réformes favorisant la poursuite des études, les administrateurs de l'Éducation nationale espèrent une démocratisation de l'enseignement. Il s'agit, en fait, de « recruter les élites sur une base démocratique ${ }^{2}$. Par ailleurs, les transformations de l'Université française s'accompagnent de changements importants dans le syndicalisme étudiant. Les syndicats étudiants s'étaient vivement opposés à la guerre d’Algérie. Avec la fin de celle-

\footnotetext{
${ }^{1}$ Ce texte est une reprise d'un chapitre de mon livre (Masson, 2008) qui présente l'histoire de la sociologie française depuis 1945 à partir de ses enquêtes empiriques.

${ }^{2}$ Pour reprendre l'expression d'Antoine Prost, dans son analyse de la politique gaullienne d'éducation (Prost, 1992, chapitre 5).
} 
ci, il leur faut trouver de nouveaux thèmes pour continuer à mobiliser les étudiants et limiter une baisse du nombre d'étudiants syndiqués. Ils vont alors davantage centrer leurs revendications sur la « condition étudiante ». Un troisième élément du contexte renvoie aux analyses sociologiques en vogue dans les années soixante sur le développement des moyens de communication de masse (la radio, la télévision, le cinéma). Des sociologues, comme Edgar Morin, pensent voir dans ce développement, l'apparition d'une « culture de masse ». L'ouvrage de Bourdieu et Passeron prend à contre-pied ces analyses qui seront partiellement remises en cause dans la seconde moitié des années soixante avec le changement de conjoncture politique ${ }^{3}$. Le contexte social et politique, lors de la parution de l'ouvrage, est donc très favorable à sa diffusion : débats publics sur l'université, revendications étudiantes et militantisme actif des étudiants. L'ouvrage va être utilisé par les organisations syndicales étudiantes et les partis politiques de gauche, à partir de la fin des années soixante, à des fins de justifications de leurs propres analyses de l'institution scolaire. Ces éléments contribueront à sa notoriété mais aussi aux interprétations ultérieures du livre.

\section{Les auteurs}

Pierre Bourdieu, né à Denguin en 1930, d'un père facteur devenu receveur distributeur des postes à Lasseube (près de Pau) dans les Pyrénées-Atlantiques est originaire de la petite bourgeoisie. Après son baccalauréat, il entra, comme Passeron, à l'École normale supérieure où il prépara avec succès l'agrégation de philosophie. A la différence des sociologues français de la génération précédente, l'un et l'autre bénéficient d'une solide formation en philosophie qui contribuera, de façon décisive, à l'orientation de leurs travaux ultérieurs. Les années cinquante avaient été marquées par la domination dans les débats intellectuels et philosophiques de la phénoménologie et de l'existentialisme dont le représentant le plus visible était Jean-Paul Sartre. Nul doute,

\footnotetext{
${ }^{3}$ Pour une analyse détaillée de ce contexte, voir Masson, 2001. Plus largement, sur ce livre, on lira avec intérêt, les contributions réunies dans Chapoulie, Kourchid, Robert et Sohn, (dir.), 2005, p. 13-119.
} 
comme il l'a souvent affirmé, que Sartre constituait pour Pierre Bourdieu un modèle négatif dans la première partie de sa carrière universitaire, rejetant chez celui-ci non seulement la philosophie du sujet et de la conscience (un « humanisme ») qu'il proposait, mais aussi son modèle d'engagement dans les débats publics. Parallèlement, une histoire de la philosophie (autour de Martial Guéroult, Jean Hypolyte) et une philosophie des sciences (Gaston Bachelard, Georges Canguilhem et Alexandre Koyré) se développaient en marge de ce courant dominant dans la philosophie française. Ces derniers envisageaient l'histoire du savoir scientifique comme une série cumulative de modèles rationnels échappant à toute représentation subjective. Cette philosophie des sciences inspire une partie importante des analyses des sciences sociales à partir de la fin des années cinquante. Elle marque un nombre assez important d'étudiants de la fin des années cinquante comme ceux des années soixante qui trouvent chez ces auteurs un nouveau modèle de scientificité pour les sciences sociales. L'anthropologie de Claude Lévi-Strauss représentait, par contre, un modèle positif. Celui-ci cherchait à dégager «la structure fondamentale de l'esprit humain ", souhaitant montrer que toute représentation du monde met en oeuvre des structures mentales inconscientes communes à des peuples différents. La carrière de Lévi-Strauss (né en 1908) dont la légitimité était basée sur une reconnaissance scientifique, élu au Collège de France en 1959 (un an après la publication de son livre Anthropologie structurale et quatre ans après le succès de Tristes tropiques), pouvait représenter un modèle à suivre pour la nouvelle génération de chercheurs en sciences sociales. C'est d'ailleurs dans cette discipline à la mode que Bourdieu commença ses premières recherches en Algérie [Bourdieu, Darbel, 1963 ; Bourdieu, Sayad, 1964].

Jean-Claude Passeron, né en 1930, dans les Bouches-du-Rhône, d'un père instituteur succéda à Bourdieu en 1961 au poste d'assistant d'Aron à la Sorbonne, après le départ de celui-là pour la Faculté de Lille. Sa collaboration avec Bourdieu va durer jusqu'en 1972. Les premiers travaux de Passeron, ceux qu'il réalise seul, porte sur la culture : il mène ainsi une enquête sur l'usage de la photographie parmi le personnel des usines Renault au début des années 1960 [Passeron, 1962] ${ }^{4}$. Après 1972, il continue

\footnotetext{
${ }^{4}$ Cette première recherche de Passeron repose sur une enquête par questionnaire et des entretiens auprès
} 
d'ailleurs dans ce domaine avec des recherches sur la lecture, l'usage de la documentation audio-visuelle dans les bibliothèques, les artistes (en collaboration avec Raymonde Moulin) ainsi qu'une critique du rapport des sociologues (et de leurs analyses, en particulier celles de Bourdieu) à la culture populaire [Grignon, Passeron, 1989]. La soutenance d'une thèse d'État à l'Université de Nantes en 1980 ouvre une nouvelle période sur des travaux d'épistémologie de la sociologie qui aboutissent, en particulier, à la publication du Raisonnement sociologique [Passeron, 1991]. L'ouvrage prend le contrepied de celui qu'il avait signé vingt ans plus tôt avec Bourdieu et Chamboredon [Bourdieu, Chamboredon, Passeron, 1968].

\section{Les Héritiers : à l'initiative de nouvelles enquêtes de sociologie de l'éducation}

On dit souvent que c'est avec Les Héritiers que les intellectuels et les politiques ont découvert les inégalités sociales à l'école. C'est exagéré. Il y avait eu des travaux, dans les années 1950, par des chercheurs de l'INED sur les inégalités d'orientation entre les différentes filières de l'institution scolaire. Mais on les rapportait à des inégalités d'ordre économique (les différences de revenus). La force du livre de Bourdieu et Passeron est de proposer une thèse simple et fondée empiriquement sur ces inégalités sociales, et en même temps, de prendre le contre-pied des théories en vogue à cette époque. Les inégalités devant l'école ne résultent pas seulement de l'inégalité des ressources économiques des familles. Elles sont aussi dans l'école, elles se produisent pendant tout le cursus scolaire et elles s'expliquent essentiellement par l'appartenance sociale, parce que les classes sociales ont des expériences différentes du monde. Les étudiants ne forment-ils pas un grand groupe social homogène? Non, s'insurgent nos deux sociologues, car les probabilités d'accès à l'enseignement supérieur sont très différentes selon les classes sociales d'origine des étudiants. Plus encore, leur rapport aux

\footnotetext{
de 44 membres du club de photos de l'entreprise Renault. L'auteur n'y examine pas seulement l'équipement et l'usage du matériel photographique des adhérents du club, mais aussi le rapport aux différents genres photographiques et les catégories de perception et de jugement dans ce domaine. La conclusion propose une analyse des fonctions sociales de l'image photographique.
} 
études est radicalement opposé et ceux originaires des classes aisées, les héritiers qui ont intégré depuis longtemps les normes culturelles de l'école (qui sont aussi celles de leur milieu social), peuvent se permettre un rapport faussement distant tout en ayant les moyens d'y réussir. Un enseignement de masse où l'orientation des élèves, à la fin d'un tronc commun, dépendrait de leurs aptitudes et de leurs mérites ne permettrait-il pas une démocratisation de l'école? Pas davantage, car il n'y a pas de «dons scolaires », l'école n'est pas libératrice. Elle contribue au maintien des inégalités et, par là, à la conservation de l'ordre social.

Les Héritiers a radicalement changé la vision que l'on avait à l'époque de l'école. Auparavant, les analyses (sociologiques ou non) sur l'institution scolaire faisaient preuve de sympathie à l'égard de celle-ci. A partir de la fin des années soixante, de nouvelles analyses proposent une image plus sombre de cette institution en insistant sur ses fonctions de reproduction des inégalités ${ }^{5}$. Cela signifie qu'avec Les Héritiers se constitue progressivement une perspective autonome des sciences sociales sur l'école, qui est bien sûr le fruit de la conjoncture de l'époque, mais qui montre que les recherches empiriques peuvent apporter des innovations théoriques ou, plus précisément, élaborer un point de vue différent des débats d'époque tout en partant de ceux-ci. Les Héritiers fournit aussi un exemple, parmi d'autres, d'un ouvrage dont le succès tient surtout à la manière dont celui-ci fut interprété et utilisé par la suite, selon des contextes variés. Il faut simplement rappeler ici que Les Héritiers ignorait la dimension temporelle du phénomène étudié. Les auteurs laissaient de côté toute analyse diachronique des parts respectives des différentes catégories socioprofessionnelles dans l'enseignement supérieur pour conclure à l'intangibilité des inégalités sociales face à la culture. Ils ne s'intéressaient pas à la forte croissance des effectifs de l'enseignement supérieur. Mais, plus encore, le succès public du livre et ses diverses utilisations eurent pour effet d'étendre le domaine de pertinence des Héritiers à une base plus large que le sujet sur lequel il portait initialement. En effet, l'ouvrage porte sur les inégalités des étudiants face à la culture comme le suggère d'ailleurs son sous-titre. Les données empiriques du livre, comme celles du rapport dont il est issu, confirment qu'il s'agit bien là du sujet traité. Cependant, au fil du

\footnotetext{
5 J'ai examiné le cadre de la réception de ce livre qui a contribué à ce retournement dans [Masson, 2005].
} 
temps, l'ouvrage est utilisé comme un livre qui traite des inégalités sociales dans l'enseignement et des principes par lesquels l'institution scolaire favorise le maintien de ces inégalités. Plusieurs enquêtes sur la culture ou l'école dirigées par Bourdieu et Passeron allaient dans ce sens et d'autres enquêtes des mêmes auteurs sur des sujets voisins (l'art, la photographie) confirmaient l'importance de la classe sociale comme facteur décisif pour comprendre les pratiques dans ces autres domaines.

\section{Chronologie des enquêtes françaises sur les inégalités devant l'école entre 1950 et 1973} Les premières enquêtes sont réalisées par l'Institut National des Études Démographiques (INED) à partir de 1944 dans le cadre de la Fondation pour l'étude des problèmes humains, mais les comptes rendus sont plus tardifs.

1950 : G. Heuyer, H. Pieron, A. Sauvy, Le niveau intellectuel des enfants d'âge scolaire. Un enquête nationale dans l'enseignement primaire, tome 1 , Cahiers nº 13 , INED.

1954 : H. Bastide, H. Bergues R. Gille, A. Girard, L. Henry, J. Sutter, L. Tabah, tome 2 : La détermination des aptitudes. L'influence des facteurs constitutionnels, familiaux et sociaux, Cahiers $n^{\circ} 23$, INED.

1952 à 1955 : Plusieurs articles dans Population, par A. Girard et al., sur l'orientation à la fin des études primaires.

1959 : P. Naville (dir.), Ecole et société, Paris, Librairie Rivière.

1963 à 1965 : Enquête de Girard et al., sur les élèves quittant le Cours Moyen $2^{\mathrm{e}}$ année. Plusieurs articles dans la revue Population.

1964 : P. Bourdieu et J.-C. Passeron, Les Héritiers, Edition de Minuit (enquête commencée en 1961).

1965: P. Bourdieu P., Passeron J.-C., Saint-Martin M., Rapport pédagogique et communication, Mouton.

1968 : Numéro double de la Revue française de sociologie, codirigé par P. Bourdieu et V. Isambert-Jamati.

1970 : P. Bourdieu, J.-C. Passeron, La Reproduction, Paris, Edition de Minuit.

1970 : A. Girard et al., Population et l'enseignement, Paris, Presses Universitaires de France. (Cet ouvrage est un recueil des articles parus dans la revue Population).

1971 : C. Baudelot et R. Establet, L'école capitaliste en France, Paris, Maspéro.

1973 ; R. Boudon, L'inégalité des chances, Paris, Armand Colin.

La perspective de Bourdieu et Passeron constituera ultérieurement un des thèmes récurrents de la littérature sociologique sur les étudiants. En effet, les ouvrages français qui paraissent ensuite, et qui prennent pour objet les étudiants, se situeront 
généralement par rapport à cette problématique. Ainsi, le livre Les étudiants, l'emploi et la crise, publié en 1981 par Christian Baudelot, Roger Benoliel, Hubert Cukrowicz et Roger Establet présente une analyse opposée (de façon implicite) aux Héritiers. Les étudiants sont présentés comme un groupe social homogène en raison des fonctions que les auteurs assignent à l'Université. Celle-ci produit des « petits bourgeois » chargés d'assurer des fonctions d'encadrement dont la société a besoin. II faudra ensuite attendre une dizaine d'années (1992) avant que les étudiants redeviennent un objet d'enquête empirique à l'occasion de la forte croissance des effectifs dans l'Université. Pour autant, la question de l'homogénéité ou non des étudiants comme groupe social restera, jusqu'à une date récente, un fil conducteur de l'ensemble des enquêtes empiriques portant sur cette population.

Au-delà de ce thème de recherche, le livre de Bourdieu et Passeron est un point de référence de travaux ultérieurs en sociologie de l'éducation. Le fait que Les Héritiers soit parfois associé à La Reproduction à partir de 1970 contribua aussi à sa notoriété, le premier livre apparaissant a posteriori comme une illustration empirique des thèses présentées dans le second. La sociologie de l'éducation devient alors, dans les années 1960, une spécialité importante de la sociologie française, à laquelle se rattachent de plus en plus d'enquêtes empiriques. Le thème principal, voire unique, des recherches est d'évaluer le rôle de l'école dans la construction des inégalités sociales, de saisir quel est le lien entre l'école et les classes sociales. La question est donc celle du lien entre la formation scolaire et les positions sociales acquises ultérieurement (après l'accès aux emplois). Un thème qui resta dominant jusqu'au début des années 1980. Les sociologues français conservent jusqu'à aujourd'hui un intérêt pour les inégalités scolaires, le fil rouge de toute la sociologie de l'éducation. L'attachement aux valeurs de l'idéal républicain (l'égalité) et les débats récurrents qui y sont associés rendent ce domaine de la sociologie inévitablement lié, sous des formes variées, à cette question politique qu'est l'inégalité des chances. Si l'analyse des inégalités reste donc centrale dans la sociologie française des années 1990-2000, le développement des recherches ethnographiques conduira à un déplacement de la perspective. La construction des inégalités à l'intérieur de l'école deviendra alors un objet fréquent de recherches. 
La position extrême, ou attribuée comme telle (car le point de vue de Bourdieu et Passeron sur l'école est plus complexe que la vulgate qui s'est progressivement imposée à partir du titre de leur livre de 1970, La Reproduction) ${ }^{6}$, de leurs analyses est une des raisons de la notoriété de celles-ci. En effet, Les Héritiers et La Reproduction contiennent des critiques de toutes les analyses sur l'Université en vogue en France dans les années soixante. Ainsi, l'interprétation technocratique assez proche du point de vue gouvernemental est critiquée. L'application à l'Université par Michel Crozier de son analyse du phénomène bureaucratique est aussi visée explicitement. Il en est de même des perspectives freudo-gauchistes et de l'analyse marxiste rapide et simpliste qui réduit l'Université à une université de classes simple rouage du capitalisme monopoliste d'Etat, ignorant ainsi l'autonomie de l'institution scolaire mise en évidence par Bourdieu et Passeron. Par-là, les deux ouvrages de ces auteurs (dans ce domaine) devenaient des points de repère ou de référence pour la constitution de nouvelles analyses alternatives. De fait, l'ouvrage de Baudelot et Establet, L'école capitaliste en France (1971), d'une part, et celui de Boudon, L'inégalité des chances (1973), d'autre part, peuvent être - et ont parfois été - lus comme des analyses alternatives. Les comptes rendus de ce dernier livre et, ultérieurement, de nombreux manuels de sociologie destinés aux étudiants ont construit une opposition binaire entre Les Héritiers et L'inégalité des chances ${ }^{7}$. Cette opposition binaire a été utilisée, pendant un certain temps, comme un "moyen d'orientation » dans la profession des sociologues, comme l'est aussi la notion « d'école » pour qualifier une tradition de recherche ou un ensemble de travaux d'auteurs divers placés sous une même bannière théorique.

\footnotetext{
${ }^{6}$ Pour Bourdieu, voir, par exemple, [Bourdieu, 1997] et pour Passeron, son examen critique de cette thèse dans [Passeron, 1986 ; Passeron, 2002].

${ }^{7}$ Cela ne signifie pas qu'il n'existe pas de différences réelles entre Les Héritiers et L'inégalité des chances sur la question des inégalités sociales de scolarisation. Une des différences majeures réside dans le fait que Raymond Boudon minimise l'importance de l'héritage culturel dans l'explication de ces inégalités et accorde, implicitement, une place prépondérante aux inégalités économiques comme facteur les déterminant. Il rejoint par là les explications avancées antérieurement dans les travaux d’Alain Girard.
} 


\section{L’innovation méthodologique et rédactionnelle}

A s'en tenir au mode de recueil des données, le livre ne diffère pas des monographies de sociologie du travail réalisées dans la seconde moitié des années cinquante. La documentation recueillie et présentée repose surtout sur des entretiens et l'exploitation de questionnaires. La démarche statistique apparaît comme le principal instrument de preuve. Où réside alors l'innovation? Une innovation technique (ou méthodologique) a été immédiatement perçue à l'époque: il s'agit des tableaux et graphiques (du chapitre 1 du livre Les Héritiers) qui mesurent les chances objectives d'accès (ou probabilités d'accès) à l'enseignement supérieur selon les différentes catégories socioprofessionnelles. Il s'agissait d'une manière frappante et objective de mesurer les inégalités. Les enfants de professions libérales et cadres supérieurs avaient ainsi près de 42 fois plus de chances d'accéder à l'Université que les enfants d'ouvriers $(58,5 / 1,4)$. Le livre introduit aussi des innovations importantes sur le plan de l'analyse des données. Les comptes rendus de recherches sociologiques des années cinquante conservaient un caractère très descriptif. Les réponses des personnes interrogées étaient tenues pour être une expression fidèle de leur jugement ou de leur comportement. Bourdieu manifestait une claire hostilité pour ces recherches empiriques qu'il jugeait " positivistes ", dénuées à ses yeux d'ambition théorique. Dans Les héritiers, les extraits d'entretien sont présentés pour illustrer une pratique par leur contenu informatif immédiat, ou pour illustrer un comportement effectif contraire aux déclarations. Pour Bourdieu et Passeron, il n'y a pas de questions et de réponses neutres et il ne faut pas croire qu'un questionnaire garantit l'univocité des réponses. II n'existe donc pas de données immédiatement accessibles, elles sont toujours construites par le chercheur.

Plus encore, le livre repose à la fois sur la philosophie des sciences qui se développait à l'époque (en s'appuyant sur l'idée qu'il fallait rompre avec le sens commun) et sur le modèle de l'anthropologie structurale dans la recherche de « lois générales » des comportements sociaux. La détermination objective des «conditions sociales de possibilités » des comportements (une notion qui trouve directement son inspiration dans la philosophie de Kant, en particulier telle qu'elle est exprimée dans la Critique de la raison pure, qui restait une référence) contribue aussi à définir un modèle de scientificité 
pour la sociologie. Les comportements sociaux sont en effet ainsi déterminés par des éléments structurels dont les individus n'ont pas nécessairement conscience. La fonction de l'école dans la société (qui est de reproduire l'ordre social) est présentée comme un invariant qui permet d'expliquer les raisons pour lesquelles les inégalités scolaires se perpétuent. Pour Bourdieu et Passeron, il s'agit alors d'établir une sociologie scientifique sur le modèle des sciences de la nature et, plus précisément, des sciences physiques. Sur ces points, ce livre s'opposait à la philosophie existentialiste (comme celle de Sartre) nettement dominante depuis une quinzaine d'années. Finalement, Les Héritiers et l'ensemble des travaux réalisés sous la direction de Bourdieu et Passeron renouvelaient ce que pouvait proposer la sociologie: des analyses jugées rigoureuses et scientifiques (parce que s'appuyant principalement sur l'outil statistique) mais conceptualisées (avec de nombreux concepts comme celui d'éthos de classe, par exemple; il suffit de se reporter à l'index de l'ouvrage) et qui prétendaient analyser de façon subtile les comportements.

\section{L'influence diffuse : les normes d'une nouvelle définition de la sociologie}

Le rôle des Héritiers dans l'histoire de la sociologie française ne se réduit pas à cet aspect. L'ouvrage a participé à la redéfinition de la sociologie dans les années soixante. En premier lieu, Les Héritiers apparaît comme un modèle de compte rendu empirique qui associe à la fois - c'est cette combinaison qui est essentielle - l'usage de statistiques obtenues par des sources variées (fichiers administratifs et enquêtes par questionnaire), ce qui est à l'époque le signe d'une enquête scientifique, l'interprétation non positiviste des réponses des enquêtés (c'est-à-dire ne pas prendre les réponses de ceux-ci à leur valeur faciale) et l'usage de catégories d'analyse abstraites. Les Héritiers apparaissait donc comme un modèle d'enquête empirique pour les nouvelles générations d'enseignants chercheurs et de chercheurs qui débutèrent leur carrière dans les années soixante. De ce point de vue, Les Héritiers est dans la lignée des travaux précédents de Pierre Bourdieu sur l'Algérie. De plus, les travaux de Bourdieu sur l'Algérie comme ceux 
sur les étudiants ou l'art, utilisent largement le code des Catégories socioprofessionnelles comme catégorie de traitement et d'analyse des données en accordant à celle-ci un rôle déterminant dans les enquêtes. Ces travaux vont ainsi avoir une influence sur la nouvelle génération de statisticiens de l'Institut National de la Statistique et des Études Économiques (INSEE) qui est à l'origine du code des Catégories socioprofessionnelles. Une partie d'entre eux a été formée par Bourdieu et certains ont collaboré directement aux enquêtes qu'il a dirigées.

En second lieu, la sociologie française des années cinquante ne reposait pas sur le triptyque des fondateurs (Marx, Durkheim, Weber) comme celle d'aujourd'hui. Les prémisses de la culture de la sociologie, telles qu'elles sont présentées par Wallerstein, n'étaient pas entièrement établies en France à cette époque [Wallerstein, 1999]. De quoi s'agit-il ? On présente souvent la sociologie comme une discipline éclatée entre plusieurs pôles que l'on qualifie parfois comme autant d'écoles, de courants différents. Ce mode de présentation est fréquent et assez pratique pour l'enseignement. II masque pourtant ce qui rassemble les sociologues, ce qui en fait une communauté professionnelle avec ses revues, ses départements universitaires, ses laboratoires, ses objets de recherche, etc. Au-delà de ces éléments institutionnels indispensables qui fondent en pratique l'homogénéité de la profession, il existe selon Wallerstein, une « culture de la sociologie », un socle commun minimal, un ensemble de prémisses qui doit être simple afin qu'il puisse être partagé par le plus grand nombre. Selon Wallerstein, la première de ces prémisses est issue de l'œuvre de Durkheim. Elle insiste sur la réalité objective des faits sociaux et elle peut être résumée par la formulation suivante : «il existe des groupes sociaux qui ont des structures explicables, rationnelles »(p.165). Ce postulat est étroitement associé à l'idée que les faits sociaux doivent être traités comme des choses, pour reprendre la formule de Durkheim. Si ce premier postulat, qui permet de fonder la sociologie comme science, était partagé par une grande partie des sociologues français des années cinquante, cette dernière formulation durkheimienne, issue des Règles de la méthode sociologique, n'était pas reprise par les sociologues français. Raymond Aron avait, par exemple, un avis réservé et sceptique sur l'œuvre de Durkheim, y compris Les Règles de la méthode sociologique. La deuxième prémisse, tirée de l'œuvre de Marx, repose sur la 
pérennité du conflit social. La troisième, issue de l'œuvre de Weber, repose sur l'existence de mécanismes de légitimation pour contenir ce conflit social. Les notions de «légitimité » et de «domination légitime » sont évidemment ici essentielles. Weber était largement ignoré, mais pas méconnu, avant les années soixante, en particulier par les sociologues qui réalisaient des enquêtes empiriques.

Les recherches empiriques menées par les sociologues français entre 1945 et la fin des années cinquante ne reposent jamais sur ces trois prémisses à la fois. Elles ne prennent pas non plus appui sur les schèmes généraux développés par ces trois « pères fondateurs ». Si considérer les faits sociaux comme objectivables n'était pas rare dans une période où l'objectif de connaissance de la réalité sociale était essentiel, l'idée de centrer, par exemple, les analyses sur les phénomènes sociaux à partir de l'existence de mécanismes de domination n'était pas fréquente. Les ouvrages de Weber n'étaient pas disponibles en français à cette époque et Aron, qui avait été l'un des premiers en France à présenter le sociologue allemand dès 1935, n’avait pas réellement emporté l'adhésion. La comparaison avec les Etats-Unis est d'ailleurs éclairante. Comme le souligne Connell [Connell, 1997] pour la sociologie américaine, voir en Durkheim, Marx et Weber les « trois pères fondateurs » de la sociologie est relativement récent. C'est au cours des années 1960 en France, et pendant la décennie précédente aux Etats-Unis, que l'on voit apparaître cette organisation de la sociologie autour des «trois pères fondateurs ». Elle correspond à la nécessité d'une définition spécifique de cette discipline à un moment où elle s'institutionnalise progressivement face aux disciplines concurrentes dans un contexte de forte croissance du nombre d'étudiants. Une pédagogie centrée sur la lecture des textes canoniques de ces trois fondateurs se développe aussi à cette période. Parmi les premières présentations en France d'une définition de la sociologie qui s'appuie conjointement sur Durkheim, Marx et Weber on trouve celle d'Aron dans ses cours professés à la Sorbonne dans les années 1960-1962 mais publiés en 1967. L'ouvrage d'Aron [Aron, 1967] est d'ailleurs le premier à présenter en France une histoire de la sociologie à partir de l'histoire des théories des auteurs du XIX ${ }^{\mathrm{e}}$ siècle. Cependant, Aron analyse bien d'autres auteurs que Durkheim, Marx et Weber; il évoque ainsi Montesquieu, Comte, Pareto, et Tocqueville. Pour quelles raisons ces derniers n'ont-ils 
pas été largement réutilisés par la suite au profit d'une focalisation autour de Durkheim, Marx et Weber? Le contexte intellectuel de la discipline est probablement l'une des raisons majeures de cette relative mise à l'écart. En effet, on ne peut pas s'étonner du relatif désintérêt pour Tocqueville dans une période marquée par le structuralisme et, à partir de la fin des années soixante, par un certain regain du marxisme en particulier à travers les lectures d'Althusser de l'œuvre de Marx. Tocqueville sera plus fréquemment présenté comme un «fondateur » de la sociologie au cours des années 1970, et encore pas de manière systématique. Pareto était davantage perçu comme un économiste et Montesquieu comme un philosophe politique. Comte était jugé comme le représentant du positivisme moins directement utile pour les études empiriques.

C'est principalement le contexte intellectuel qui définit quels sont les «pères fondateurs » de la discipline et non une définition à partir des œuvres de ceux qui sont qualifiés de sociologues antérieurement. La présentation de Raymond Aron restait relativement abstraite, elle ne pouvait suffire pour asseoir la sociologie face aux autres disciplines concurrentes. La sociologie devait pouvoir s'appuyer sur des analyses empiriques d'objets circonstanciés. Les Héritiers est le premier livre à base empirique dans la sociologie française qui remplit cette fonction en s'appuyant sur les trois fondateurs (Durkheim, Marx, Weber) pour analyser l'institution scolaire. Considérant que les pratiques étudiantes ainsi que le rapport aux études universitaires pouvaient être objectivées par la démarche sociologique, Bourdieu et Passeron souhaitaient montrer que l'institution scolaire avait principalement pour conséquence de légitimer les inégalités culturelles, de maintenir ainsi les privilèges des catégories sociales les plus aisées ; autrement dit de maintenir l'ordre établi. Ce qui me paraît essentiel ici, ce n'est pas tant la thèse elle-même que le fait que l'analyse soit fondée sur les trois axiomes évoqués précédemment et qu’il soit possible de les retrouver dans la plupart des analyses sociologiques sur l'institution scolaire à partir des années 1960, que celles-ci adoptent ou non la thèse défendue par Bourdieu et Passeron. Par rapport à leurs devanciers, ces auteurs sont parmi les premiers à proposer une analyse d'un phénomène social non seulement basée sur une approche plus globale des rapports de classe (en s'inspirant de Marx) mais aussi sur certains des principes de légitimation (Weber) qui fonderaient, selon 
eux, l'ordre établi et la stabilité de ces rapports, objectivables par des données quantitatives (Durkheim) ${ }^{8}$. Dès lors, ils font ainsi de l'établissement de relations stables pour un ordre de phénomènes donné, associé à l'intention de dégager une ou quelques caractéristiques essentielles de cet ordre, un élément central de la définition de la sociologie à cette période.

\section{La fonction de dévoilement des structures sociales}

On a dit parfois que Bourdieu avait changé de point de vue envers la question de l'engagement du sociologue dans la Cité dans la seconde moitié des années 1980. Il s'était mis à intervenir plus fréquemment dans les débats publics et sur la scène politique alors qu'il aurait fait preuve d'une distanciation plus grande dans la première partie de sa carrière. De fait, on trouvera aisément des textes de Bourdieu qui prônent une neutralité axiologique nécessaire au travail scientifique et des entretiens où il s'explique sur ce changement d'attitude. D'un autre côté, ses épigones (en particulier les plus récents) aiment souligner que la sociologie de Bourdieu a toujours été engagée, comme l'illustrerait le recueil de ses interventions qualifiées de politiques [Bourdieu, 2002]. II est probablement vain de vouloir écrire une histoire à l'aune des attitudes les plus récentes, de chercher une ligne directrice dans ce domaine (ou dans d'autres), une flèche ou un sens de l'histoire. C'est une lecture intellectualiste (il aurait sans doute dit scolastique) des œuvres littéraires et de sciences sociales alors qu'on y trouve inévitablement inflexions, contradictions, directions inexploitées ou abandonnées. Une telle manière d'écrire ou de présenter l'histoire fait comme si il y avait toujours une visée intentionnelle dans le rapport à la politique alors que, comme chacun le sait d'expérience, les événements peuvent parfois nous contraindre à l'engagement. La Guerre d'Algérie était un de ces moments où il était difficile de ne pas prendre position, d'autant plus quand on

\footnotetext{
${ }^{8}$ Les notions de Durkheim et de Weber utilisées dans l'ouvrage occupent une place de choix dans l'index. Ce qui est, là aussi, une originalité pour l'époque.
} 
y était en poste, comme l'illustrent d'autres cas de chercheurs en sciences sociales à l'époque?.

Là n'est pourtant pas l'essentiel. Les recherches de Pierre Bourdieu (et de son équipe) illustrèrent (et continuent d'illustrer) une double caractéristique ancienne de la sociologie, celle du dévoilement et de la dénaturalisation du social, que l'on trouve aussi chez Durkheim, par exemple. Comme le souligne Peter Berger, la sociologie a dans ses principes un projet de démystification des systèmes sociaux qu'elle étudie [Berger, 2006, p. 72-79]. Nous ne prenons pas ce que les institutions disent d'elles mêmes pour argent comptant. Nous voulons alors aller voir ce qui se cache derrière la façade, la vitrine officielle. Comme nous étudions toujours des pratiques, des phénomènes qui dépassent le cadre officiel de ceux-ci, alors nous dévoilons inévitablement des ressorts cachés de l'action. L'une des manières de le faire est de dire que les institutions remplissent des fonctions différentes de celles qu'elles s'assignent officiellement. Ainsi, l'école ne fait pas qu'instruire mais elle contribuerait au maintien des inégalités sociales, à la socialisation des individus.

Les recherches empiriques de Pierre Bourdieu ont toutes pour point commun de placer les classes sociales au centre du recueil des données et de l'analyse. Pour chacun des domaines auquel il s'est intéressé (l'art, l'éducation, la culture, la politique), il a montré que l'appartenance sociale organisait les pratiques. Sa grande enquête sur le goût, La distinction. Critique sociale du jugement (1979), illustre parfaitement ce souci. Le thème de la distinction comme mécanisme de différenciation sociale et le terme lui-même (de distinction) n'étaient évidemment pas nouveaux. Ils sont au cœur du livre d'Edmond Goblot, La barrière et le niveau (1925) qui avait été réédité en 1967. Bourdieu reprend donc ici le thème en lui donnant une consistance empirique plus grande et plus large et dans des domaines plus variés. Si il y avait un fil directeur à l'œuvre de Bourdieu, c'est incontestablement celui-ci qu'il faudrait retenir. Bourdieu veut montrer dans cette recherche que nos pratiques individuelles en matière alimentaire, vestimentaire,

\footnotetext{
${ }^{9}$ Dans [Bourdieu, 2002], on notera d'ailleurs la disproportion entre la part des textes rassemblés pour la période avant 1980 et ceux qui concernent la période suivante. Au moins peut-on conclure que la publication par Bourdieu de textes « engagés » dans des supports de publication non académiques devient plus fréquente, ce qui ne dit évidemment rien de l'engagement antérieur.
} 
culturelle et politique forment un ensemble homogène défini par notre appartenance à telle ou telle classe sociale (ou fraction de classe). II n'y a pas de goût personnel mais une hiérarchie sociale des goûts définie par l'espace des classes sociales. Son analyse s'oppose ainsi à la conception, probablement courante, que le goût est une affaire de choix personnel, de libre arbitre, et qu'il ne serait donc pas socialement déterminé. En cela, elle dévoile une structure que nous ne soupçonnons pas. En même temps, elle dénaturalise notre conception du goût et, plus largement, les pratiques culturelles. Audelà d'une perception courante et commune, «tous les goûts sont dans la nature; les goûts et les couleurs ne se discutent pas ", Bourdieu nous montre, comme le fait souvent la sociologie, qu'ils sont déterminés par notre appartenance sociale (sexe, génération, habitat, et surtout, classe sociale).

Dès qu'elles adoptent une analyse en termes de classes sociales, les recherches sociologiques empiriques oscillent entre deux directions. Soit elles tentent de caractériser globalement les rapports de classes, à la suite des analyses de Marx, afin de dégager un point de vue général sur la société organisé autour de cette notion ; L'école capitaliste en France (1971) de C. Baudelot et R. Establet serait à ranger dans ce groupe. Soit elles se proposent d'étudier les caractéristiques et les comportements d'une population empiriquement définie, généralement en partant des catégories statistiques de l'INSEE (le code des Catégories socioprofessionnielles, par exemple). Dans ce cas, elles s'appuient sur ces statistiques qui sont présentées comme des preuves à leurs analyses. Bien sûr, les recherches empiriques peuvent combiner ces deux directions en tentant d'établir une correspondance entre le code des catégories socioprofessionnelles et une analyse en termes de classes sociales, un point de vue abstrait sur les relations de domination entre les classes ; elles peuvent proposer une analyse des rapports de classes tout en souhaitant décrire les comportements typiques des principales classes sociales retenues. Les Héritiers ainsi que La distinction sont une même tentative pour concilier ces deux directions. Ces deux ouvrages, le premier publié en 1964, le second en 1979, marquèrent une période où prédominèrent deux caractéristiques : 1) la suprématie de l'analyse multivariée, basée sur des enquêtes par questionnaires et l'analyse secondaire de données ; 2) une forme de structuralisme où les sociologues prétendaient ramener 
l'ensemble des phénomènes qu'ils observaient à un ordre stable et défini principalement organisé autour d'une catégorie (les classes sociales). Le livre de Bourdieu et Passeron, Les Héritiers, remplit parfaitement cet objectif et il est ainsi devenu emblématique de cette période de la sociologie française.

\section{Références bibliographies}

BAUDELOT, Christian; BENOLIEL, Roger; CUKROWICZ, Hubert; ESTABLET, Roger. Les étudiants, l'emploi et la crise. Paris: Éditions Maspero, 1981.

BAUDELOT, Christian; ESTABLET, Roger. L'école capitaliste en France. Paris: Éditions Maspero, 1971.

BERGER. Peter. Invitation à la sociologie. Paris: La Découverte, 2006.

BOUDON, Raymond. L'inégalité des chances. Paris: Armand Colin, 1973.

BOURDIEU, Pierre. Interventions (1961-2001). Science sociale et action politique. Marseille: Agone, 2002.

BOURDIEU, Pierre. La distinction. Critique social du jugement. Paris: Seuil, 1979.

BOURDIEU, Pierre. Méditations pascaliennes. Paris: Seuil, 1997.

BOURDIEU, Pierre.; CHAMBOREDON, Jean-Claude; PASSERON, Jean-Claude. Le métier de sociologue. Paris: Mouton/Bordas, 1968.

BOURDIEU, Pierre; DARBEL, Alain. Travail et travailleurs en Algérie. Paris: Mouton, 1963. 
BOURDIEU, Pierre; PASSERON, Jean-Claude. La reproduction. Eléments pour une théorie du système d'enseignement. Paris: Éditions de Minuit, 1970.

BOURDIEU, Pierre; SAYAD, Abdelmalek. Le déracinement. La crise de l'agriculture traditionnelle en Algérie. Paris: Minuit, 1964.

CHAPOULIE, Jean-Michel; KOURCHID, Olivier; ROBERT, Jean-Louis; SOHN, Anne-Marie (dir.). Sociologies et sociologues. La France des années 1960. Paris: L'Harmattan, 2005.

CONNEL, Raewyn. «Why is classical theory classical?», American Journal of Sociology, vol. 102, n 6, 1997, p. 1511-1557.

CROZIER, Michel. Le Phénomène bureaucratique. Paris: Seuil, 1964.

FORNEL, Michel de; PASSERON, Jean-Claude (dir.). L'Argumentation. Preuve et persuasion. Paris: Éd. de l’EHESS («Enquête» 2), 2002, 191 p.

GOBLOT, Edmond. La barrière et le niveau, étude sociologique sur la bourgeoisie française moderne. Paris: PUF, 1925.

GRIGNON, Claude; PASSERON, Jean-Claude. Le savant et le populaire: misérabilisme et populisme en sociologie et en littérature. Paris: Seuil/Gallimard, École des hautes études, 1989.

LÉVI-STRAUSS, Claude. Anthropologie structurale. Paris: Plon, 1958.

LÉVI-STRAUSS, Claude. Tristes Tropiques. Paris: Plon, 1955.

MASSON, Philippe. «La fabrication des Héritiers», Revue française de sociologie, vol. 42, $\mathrm{n}^{\circ}$ 3, 2001, p. 477-507.

MASSON, Philippe. «Premières réceptions et diffusions des Héritiers (1964-1973)», Revue d'histoire des sciences humaines, $n^{\circ} 13,2005$, p. 69-98.

MASSON, Philippe. Faire de la sociologie. Les grandes enquêtes françaises depuis 1945. Paris: La Découverte, 2008.

MORIN, Edgar. L'esprit du temps. Paris: Grasset, 1962.

PASSERON, Jean-Claude. «La signification des théories de la reproduction socioculturelle». Revue internationale des sciences sociales, $n^{\circ} 110,1986$, p. 127-138; p. 619-626; p. $665-675$.

PASSERON, Jean-Claude. La photographie parmi le personnel des usines Renault. Paris: CSE, ronéotype, 1962. 
PASSERON, Jean-Claude. Le raisonnement sociologique. L'espace non poppérien du raisonnement naturel. Paris: Nathan, 1991.

PROST, Antoine. Éducation, société et politiques. Une histoire de l'enseignement en France de 1945 à nos jours. Paris: Seuil, 1992.

WALLERSTEIN, Immanuel. «L'héritage de la sociologie, la promesse de la science sociale». Sociétés contemporaines, $n^{\circ}$ 33-34, 1999, p. 159-194.

Recebido em: 10/10/2013 Aprovado em: 28/02/2014

Universidade do Estado de Santa Catarina - UDESC Programa de Pós-Graduação em Educação - PPGE Revista Linhas Volume 15 - Número 29 - Ano 2014 revistalinhas@gmail.com 